\title{
El gobierno digital y su implementación en el estado
}

\author{
Dra. Catherine Olive Vargas Bravo \\ Cathy-9608@ hotmail.com \\ Universidad César Vallejo \\ Lima-Perú
}

\section{RESUMEN}

El presente trabajo tiene por finalidad dar a conocer, que la implementación del gobierno digital en el estado resulta necesaria, puesto que forma parte significativa de su transformación. Es decir, su puesta en marcha tiene como propósito que las instituciones del gobierno logren adaptarse a los diversos cambios que ocurren en la sociedad, y también para lograr mantener una constante transparencia del sector público con los ciudadanos en todos los servicios que brinda a través de su gestión pública. Por otra parte, se señala que este factor se vincula directamente con muchos sectores, entre ellos resaltamos el sector educación, el cual debido a la situación por la que atravesamos actualmente se ha visto bastante expuesto. Asimismo, se precisa que el sector educación se encuentra en una etapa de renovación constante, es decir, la gestión de educación debe ser capaz de adaptarse a todo tipo de circunstancias que se puedan presentar y el empleo del gobierno digital es un buen inicio.

Palabras claves: gobierno digital; gobierno electrónico; implementación en el estado. 


\title{
The digital government and its implementation in the state
}

\begin{abstract}
The purpose of this paper is to show that the implementation of digital government in the state is necessary, since it is a significant part of its transformation. That is to say, its implementation is intended for government institutions to adapt to the constant changes occurring in society, and also to maintain a constant transparency of the public sector with citizens in all the services it provides through its public management. In another it is pointed out that this factor is directly linked to many sectors, among them we highlight the education sector, which due to the situation we are currently going through has been quite exposed. Also, it is specified that the education sector is in a stage of constant renewal, i.e., education management must be able to adapt to all kinds of circumstances that may arise and the use of digital government is a good start.
\end{abstract}

Keywords: digital government; e-government; implementation in the state.

Artículo recibido: 02 noviembre. 2021 Aceptado para publicación: 28 noviembre 2021 Correspondencia: Cathy-9608@ hotmail.com Conflictos de Interés: Ninguna que declarar 


\section{INTRODUCCIÓN}

Según el autor explicò que el gobierno digital se le puede definir como un mecanismo de política pública que modifica las diversas acciones del gobierno y la práctica profesional de la labor social; a su vez, determina una relación significativa entre el poder y el gobierno digital y puesta en marcha de políticas de gobierno electrónico, saltarían decisiones planificadas que mayormente copian el formato de poder capitalista representado en el código técnico del gobierno digital (Scholl, 2021).

Asimismo, el autor Cruz (2018) menciona que la gobernanza digital se relaciona con muchas aplicaciones que pueden ayudar a implementar o informar a todos los ciudadanos sobre diversos temas. Del mismo modo se menciona que hay diversos sitios web e incluso aplicaciones para los diversos dispositivos digitales, los cuales hacen que el Gobierno Digital tenga una visión amplia y una mejor gestión, ya que antiguamente esto no era posible debido a la falta de tecnología. Por esa razón que se busca es implementar el uso de las TIC mejorar los procesos y agilizar los trámites que necesitan los ciudadanos, ya que todo lo que hace y debería hacer el gobierno es para beneficiar a la sociedad en general.

En la misma visión, García y Plasencia (2020) agregan que el gobierno digital o electrónico forma parte de la transformación del estado y sociedad, ya que su fin es brindar un rápido acceso a los ciudadanos para obtener información de diversos trámites, también, brinda la transparencia y transmitir la confianza que necesita tener el Estado con el pueblo para entablar una buena relación y la participación los ciudadanos, al igual que tengan la información actualizada.

Por otro lado, Zientarski et. al (2019) precisa que cuando se habla de la educación y las reformas que el sector público puede ofrecer para cada país en el que se lleve a cabo, es indispensable pensar en un primer momento en la ideología de la "modernización", que va de la mano con el gobierno digital, puesto que sirve como medio para tratar de reivindicar los malos inicios que haya podido tener el Estado en el presente país, con el objetivo de poder brindar una educación adecuada a la población de acuerdo a las circunstancias actuales.

Por esta razón; es preciso mencionar a los autores Correa et al. (2020) hablan sobre las actividades en la gestión pública están vinculadas con el Estado, y se resalta la gran influencia interna en el sector educativo; asimismo, se crea e implementa distintas 
reformas de este sector en las últimas décadas, dentro de ellas la puesta en marcha del gobierno electrónico, con el propósito de poder tener un mejor desarrollo en los estudiantes. Igualmente, se busca que dicho sector se pueda adaptar a las problemáticas que traen consigo los constantes cambios en la sociedad.

De igual forma, en lo que respecta a dicho sector, las competencias tecnológicas aportan a disminuir las brechas digitales, para que los ciudadanos puedan tener acceso e integración en la educación para formarse como personas y obtener mejores resultados dado que, buscan, procesan y comunican información usándola como conocimientos en sus proyectos y trabajos de investigación para que sea útil en el futuro de cada persona, optimizando los servicios que se les brinda a todos (Maldonado, 2018)

Asimismo, la gestión pública del Estado ha buscado maneras de poder integrar a los estudiantes con necesidades educativas mediante el uso de competencias tecnológicas para una educación más adecuada a las necesidades que puedan presentar, puesto que tendrían más facilidades al momento de acceder a sus clases, ya que las podrían recibir de forma virtual. Por ejemplo, si el estudiante tiene problemas para movilizarse, esta implementación le sería de apoyo, proponiendo así una mejora que garantice la educación especial en los colegios, preocupándose así por aquellos estudiantes que son “incapaces de atender el sistema del gobierno" (Roberto, 2019).

En Perú, los autores Valenzuela et al. (2021) refieren que el implementar la tecnologia de informacion fue muy importante para la gobernanza digital ya que es necesario agilizar los procesos a traves del uso del internet el cual es una herramienta de gran significancia para el Estado que involucra a la ciudadania y beneficia a todos. Por otro lado las diversas plataformas digitales tienen muchos desafios pero a traves de estrategias por las autoridades se pueden erradicar y brindar nuevas implementaciones de plataformas digitales.

\section{ESTRATEGIAS METODOLÓGICAS O MATERIALES Y MÉTODOS}

La presente investigación se realizó con un enfoque cuantitativo,de acuerdo con Guedes dos Santos et al. (2017) señalo que una investigación cuantitativa se basa en la elaboración de estudios que están vinculados a datos númericos, los cuales fomentan la conjunción de detalles e información de esta manera permita corroborar teorias y/o hipótesis, asimismo se emplea el análisis estadístico. En la misma linea Tobi y Kampen (2017) señalan que esta permitió evidenciar los resultados de manera numérica y 
cuantificar los productos en aspectos estadísticos, asimismo recopilar información a manera de verificar o corroborar las hipótesis y/o teorías planteadas.

\section{Técnicas e instrumentos de recolección de datos}

Por otro lado, los datos utilizados se han originado a partir de una relación hipotéticadeductiva, la que nos permitirá verificar la credibilidad de los mismos, siendo que la verificación será de manera empírica. Para llegar a una se debe descartar o aceptar la hipótesis establecida, verificnado su credibilidad, y en caso el resultado fuera óptimo, se concluiría que hay una relación con la teoría inicial y los planteamientos del problema de la investigación (Sánchez,2019).

Así también, los datos utilizados se han originado a partir de una relación hipotéticadeductiva, siendo así que la verificación será de manera empírica por lo que las variables han sido estudiadas por la relación entre ellas, esto con la finalidad de obtener un mayor entendimiento y comprensión de las mismas en conjunto, así como de su importancia de investigación (Núñez,2017).

\section{Procedimiento}

Se realizó la búsqueda bibliográfica y sistemática de artículos científicos indexados en las siguientes bases de datos: Redalyc, Scielo y Scopus. La búsqueda y selección de los artículos inició en el período 2017 hasta el año 2021, para la investigación se decidió la selección de los artículos bajo una estructura relacionada con las variables, que consistió en realizar una búsqueda de información enfocada en el gobierno digital y su implementación en el Estado en el Perú, sus características, normativa nacional, beneficios, enfocándonos en la incidencia de una variable sobre la otra.

Es por ello que, los datos obtenidos tienen el objetivo de ser desarrollados de manera correcta en el presente trabajo, dado que son parte de una revisión bibliográfica ardua relacionada a la temática mencionada anteriormente, los criterios para su selección fueron la fecha de publicación, el contenido veraz y verificable de la información, así es como se desarrollan; esta será utilizada como sustento estadístico en el análisis que se llevará a cabo.

\section{RESULTADOS Y DISCUSIÓN}

\section{Resultados}

Los autores Toro et al. (2020) en su investigación señalan que el gobierno virtual en Colombia surgió con la implementación de nuevas políticas del Plan Nacional de 
Desarrollo por lo que decidieron implementar las TIC como política administrativa, de modo que se tenga un cambio significativo en la modernización del Estado, haciendo énfasis en los principios como la eficacia, eficiencia, responsabilidad y transparencia. Por otra parte, esto se da debido a las diferentes estrategias que se toman en consideración debido a las TIC lo cual permite llegar a más ciudadanos y mejorar las diversas estrategias de desarrollo.

Del mismo modo los autores Marulanda et al. (2017) refieren que el plan de desarrollo de tecnologias de informaciòn en diversos paises es gran significancia e importancia ya que abarca cuatro factores estratégicos los cuales son el empleo, la educación, la economía y el gobierno digital. En la estrategia de gobierno digital se debe tener un poder más eficiente y transparente, ya que las herramientas tecnológicas permiten mostrar la información detallada y su acceso es sencillo. Por lo que es necesario implementar nuevas estrategias de tecnología a fin de mejorar la calidad de vida de todos los ciudadanos.

Del mismo modo, los autores Mergel, et al. (2019) menciona que la transformación digital con el tiempo está tratando de cambiar las diversas expectativas de los ciudadanos sobre la necesidad de las administraciones públicas de ofrecer servicios digitales de alto valor en tiempo real. Impulsados por acuerdos supranacionales, por lo que los gobiernos están cambiando su modo de operación para mejorar la prestación de servicios, ser más eficientes y eficaces en sus diseños y lograr objetivos como el aumento de transparencia, interoperabilidad y satisfacción ciudadana. La transformación digital en el sector público significa nuevas formas de trabajar con las partes interesadas, construyendo nuevos marcos de prestación de servicios y creando nuevas formas de relaciones. Sin embargo, más allá de la disponibilidad de informes de consultoría, hay poca evidencia empírica sistemática sobre la forma en que las administraciones públicas están definiendo actualmente la transformación digital en sus prácticas diarias, cómo abordan los proyectos de transformación digital y cuáles son los resultados esperados. digital se utilizan indistintamente en la literatura. Del mismo modo la percepción de los expertos incluidos en nuestra muestra, el principal resultado que la transformación digital puede lograr se centra en medidas que tienen un impacto a largo plazo (56\%) o que conducen a un resultado específico deseado (34\%), en lugar de medibles, producción de hormigón (10\%). Los principales objetivos de la 
transformación digital se ven como impactos más amplios en las administraciones públicas como organización, sociedad y democracia.

Y finalmente los autores Jiménez et al. (2017) mencionaron que el gobierno digital para que se adapte a las (TIC) debieron informarse de los beneficios que tienen en muchos países ya que a través de ellos se han podido implementar diversas estrategias de mejora en cada país.

Adicionalmente, las TIC representan un factor importante para el desarrollo de la ciudadanía, debido a que se han implantado como un mecanismo de control de los gobiernos y como una "foro" para que los ciudadanos puedan expresar sus inquietudes y participar activamente en las decisiones del gobierno, que podrían catalogarse como herramientas útiles para superar problemas existentes propias de una sociedad democrática. Por otro lado, corresponde a los líderes gubernamentales implementar mecanismos de gestión pública, para brindar conocimiento e información a los ciudadanos, de modo que puedan participar de un gobierno eficaz y eficiente esto se puede conseguir a través del apoyo de la ciudadanía.

\section{Discusión}

Los autores Panagiotopoulos et al. (2019) precisan que las entidades gubernamentales deben tener en su agenda como punto importante mejorar los servicios públicos que realmente satisfagan a la sociedad y la ayuden a prosperar y desarrollarse. De esta manera es fundamental que se brinden todas las herramientas necesarias para la difusión de las tecnologías digitales, porque ayudan a que el país pueda tener una mejor gestión. Por otra parte, la teoría del valor público ha sido particularmente exitosa al proporcionar una alternativa a la narrativa de la Nueva Gestión Pública que concibe las tecnologías digitales como herramientas de eficiencia administrativa. En cambio, la gestión del valor público proporciona una visión más global para dar cuenta de las complejas transformaciones necesarias para cambiar el enfoque de la producción de servicios al cumplimiento de las expectativas y objetivos públicos. Por lo que es necesario innovar los recursos digitales para un mejor desarrollo del Estado.

Por otro lado, los autores Wang et al. (2018) refieren que debido a la corrupción y otros problemas que se han vuelto parte de los gobiernos, la tecnología reluce como un mecanismo de control, asimismo, impone exigencias a los gobiernos para desarrollar la capacidad de evaluar, responder e implementar nuevas tecnologías y procesos a favor de 
los ciudadanos por mejorar los procesos en el país. Esto ha creado desafíos para que los gobiernos se adapten a cambios rápidos, especialmente en la implementación de proyectos relacionados con las tecnologías de información. También señalan que los gobiernos se enfrentan cada vez más a desafíos que les obligan a imaginar la colaboración con actores no gubernamentales de una manera receptiva, al poder adaptarse a las demandas cambiantes del medio ambiente, especialmente en los esfuerzos relacionados con las tecnologías de la información.

Del mismo modo, los autores Gong et al. (2020) refieren que la tecnologia digital es un recurso necesario para que los gobiernos puedan tener mejores servicios y eficiencia.En muchos paises no se toma en consideracion el recurso de la tecnologia de informacion por falta de conocimiento pero se debe entender que este contexto mejora la calidad de vida,mejora el sistema del gobiero al implementar recursos digitales en beneficio de todos. Los diversos estudios garantizar que en el gobierno se propaga en oleadas con adaptaciones en diferentes elementos organizacionales, impactando todo el sistema administrativo e incluyendo cambios radicales e incrementales. En concordancia con nuestra investigación se señala que hay una relación entre la gobernanza digital lo que permite solucionar diversos problemas del Estado.

Y finalmente la autora Alva de la Selva (2020) señala que en Mèxico el gobierno toma mucho en consideracion el usar la tecnologia de informaciòn y comunicación pero la ciudadania si utilizan por lo que gracias al recurso del internet es posible acceder a la informacion sobre diversos temas de interes publico que les permita ampliar o obtener conocimiento con respecto a su participacion en los programas digitales. Por otro lado, el gobierno de México ha implantado herramientas tecnológicas porque permite a la ciudadanía una inclusión social y un trato casi directo con su gobierno, así como desarrollar nuevas estrategias que se han ido implementando poco a poco para llevar un gobierno digital, ya que se ha observado en otros países que realmente funciona.

\section{CONCLUSIÓN O CONSIDERACIONES FINALES}

Como primera conclusión, se puede determinar que la gobernanza digital permite subsanar ciertas problemáticas presentes en algunos sectores del Estado, lo cual permite el desarrollo y eficiente de estos.

Como segunda conclusión, los estados al implementar una digitalización han conseguido diversos resultados positivos, en el cual se puede resaltar el cierre de 
brechas digitales, mayor acceso a la educación y un crecimiento en la participación ciudadana.

Como tercera conclusión, el Estado peruano ha implementado la gobernanza digital, lo cual ha generado una relación con la ciudadanía de mayor confianza, siendo además este un facilitador en lo que respecta a los servicios que les son brindados.

\section{LISTA DE REFERENCIAS}

Alva de la Selva, A. (2020). Escenarios y desafíos de la ciudadanía digital en México.

Revista mexicana de ciencias políticas y sociales, 65(238). Obtenido de http://www.scielo.org.mx/scielo.php?pid=S0185-

$19182020000100081 \&$ script=sci_arttext

Baptista , R. (2019). Public policy, Special Education and schooling in Brazil. Educação $\quad$ Pesquisa, 45. https://www.scielo.br/j/ep/a/8FLTQYvVChDcF77kwPHtSww/?lang=en\&format $=\mathrm{pdf}$

Correa Ortiz, L., Toro Carcía, A., \& Gutiérrez Vargas, C. (2020). Estrategia de gobierno digital para la construcción de Estados más transparentes y proactivos. Trilogía $\begin{array}{llll}\text { Ciencia Tecnología } & \text { Sociedad, } & \text { 12(22), }\end{array}$ https://www.redalyc.org/articulo.oa?id=534367793009

Cruz Romero, R. (2018). Gobernanza digital: Un análisis de propuestas para Costa Rica. E-Ciencias de la Información, $8(1)$. https://www.scielo.sa.cr/scielo.php?pid=S1659-

41422018000100101\&script=sci_arttext

García Baluja, W., \& Plasencia Soler, J. (2020). Aspectos claves para la informatización y el gobierno electrónico. Revista Cubana de Ciencias Informáticas,, 14(3). http://scielo.sld.cu/scielo.php?script=sci_arttext\&pid=S222718992020000300124

Gong, Y., Yang, J., \& Shi, X. (2020). Towards a comprehensive understanding of digital transformation in government: Analysis of flexibility and enterprise architecture. Government Information Quarterly, 37(3). doi:https://doi.org/10.1016/j.giq.2020.101487

Guedes dos Santos, J., Lorenzini Erdmann, A., Schlindwein Meirelles, B., Marcellino de Melo Lanzoni, G., Pecini da Cunha, V., \& Ross, R. (2017). Integrating 
Quantitative and Qualitate data in mixed methods research. Texto \& contexto Enfarmagem, 26(3). doi: https://doi.org/10.1590/0104-07072017001590016

Jiménez Pitre, I., Martelo, R., \& Jaimes, J. (2017). Escuela de Gobierno basada en TIC: Determinante para la Accesibilidad e Integralidad del Empoderamiento Digital. Información tecnológica, 28(5). doi:http://dx.doi.org/10.4067/S071807642017000500010

Maldonado, M. (2019). El aula, espacio propicio para el fortalecimiento de competencias ciudadanas y tecnológicas. Sophia, 14(1), 39-50. Obtenido de https://www.redalyc.org/journal/4137/413755833004/413755833004.pdf

Marulanda Echevarry, C., Lopez Trujillo, M., \& Valencia Duque, F. (2017). It governence and management in public entites. Ad Minister(31), 75-92. http://www.scielo.org.co/scielo.php?pid=S169202792017000200075\&script=sci_abstract\&tlng=en

Mergel, I., Edelmann, N., \& Haug, N. (2019). Defining digital transformation: Results from expert interviews. Government Information Quarterly, 36(4). doi:https://doi.org/10.1016/j.giq.2019.06.002

Núñez Moscoso, J. (2017). Mixed methods in education research: towards a reflexive use. Cadernos de Pesquisa, 47(164). doi:https://doi.org/10.1590/198053143763

Panagiotopoulos , P., Klievink , B., \& Cordella, A. (2019). Public value creation in digital government. Government Information Quarterly, 36(4). doi:https://doi.org/10.1016/j.giq.2019.101421

Sànchez Flores, F. (2019). Fundamentos epistémicos de la investigación cualitativa y cuantitativa: Consensos y disensos. Revista Digital de Investigación en Docencia Universitaria, $\quad 13(1), \quad 102-122$. http://dx.doi.org/10.19083/ridu.2019.644

Scholl, H. (2021). The Digital Government Reference Library (DGRL) and its potential formative impact on Digital Government Research (DGR). Government Information Quarterly, 38(4). https://www.sciencedirect.com/science/article/abs/pii/S0740624X21000496

Tobi, H., \& Kampen, J. (2018). Research design: the methodology for interdisciplinary research framework. Quality \& Quantity, 52, 1209-1225. doi:https://doi.org/10.1007/s11135-017-0513-8 
Toro García, A., Gutiérrez Vargas, C., \& Correa Ortiz, L. (2020). Estrategia de gobierno digital para la construcción de Estados más transparentes y proactivos. Trilogía $\begin{array}{llll}\text { Ciencia Tecnología } & \text { Sociedad, }\end{array}$ https://papers.ssrn.com/sol3/papers.cfm?abstract_id=3620361

Valenzuela Fernández, L., Collantes Inga, Z., \& Durand Hipólito, E. (2021). Sobre la gobernanza digital, política digital y educación. Revista eleuthera, 22(2). http://www.scielo.org.co/scielo.php?script=sci_arttext\&pid=S201145322020000200088

Wang, C., Medaglia, R., \& Zheng, L. (2018). Towards a typology of adaptive governance in the digital government context: The role of decision-making and accountability. Government Information Quarterly, 35(2), 306-312. doi:https://doi.org/10.1016/j.giq.2017.08.003

Zientarski, C., Mendonca Menezes, H., \& De Oliveira Da Silvia, S. (2019). "Democratic Management" in public education: a paradox of the brazilian neopatrimonialist state?. The case of Ceará. EDUR. Educação em Revista, 35. https://www.scielo.br/j/edur/a/rMNDysykC6fCjw4x4JyBHGL/?lang=pt 